\title{
SETTLING-TIME IMPROVEMENT IN GLOBAL CONVERGENCE LAGRANGIAN NETWORKS
}

\author{
Acho L. \\ Centro de Investigación y Desarrollo de Tecnología Digital del IPN (CITEDI-IPN) \\ CITEDI-IPN, 2498 Roll Dr. \#757, Otay Mesa, San Diego CA, 92154, USA \\ leonardo@citedi.mx
}

Keywords: Lagrangian Networks, Global Convergence, Convex Optimization, Lyapunov Theory.

\begin{abstract}
In this brief, a modification of Lagrangian networks given in (Xia Y., 2003) is presented. This modification improves the settling time of the convergence of Lagrangian networks to a stationary point; which is the optimal solution to the nonlinear convex programming problem with linear equality constraints. This is important because, in many real-time applications where Lagrangian networks are used to find an optimal solution, such as in signal and image processing, this settling time is interpreted as the processing time. Simulation results applied to a quadratic optimization problem show that settling time is improved from about to 2000 to 20 seconds. Lyapunov theory was used to obtain our main result.
\end{abstract}

\section{INTRODUCTION}

Roughly speaking, a Lagrangian network is a dynamical system used to find the optimal solution to a nonlinear convex programming problem with linear equality constraints (for more details, see (Xia Y., 2003)). This dynamical system has simple structure and its complexity for implementation is low (Xia Y., 2003). Global convergence of a Lagrangian network has been analyzed in (Xia Y., 2003) and stated that it has not been studied before (Xia Y., 2003). So, the convergence (in time) of the solution of the Lagrangian networks to an equilibrium point (or stationary point), which, under some conditions, corresponds to the unique optimal solution to the nonlinear convex programming problem, is an important issue. In this short paper, we present how to modify it to improve the settling time convergence. Engineering applications in realtime of Lagrangian networks, and important references about it, are cited in (Xia Y., 2003). We developed simulation experiments applied to a quadratic optimization problem to show that the settling time could be improved from about to 2000 to 20 seconds. Lyapunov theory is employed to prove our main result.

\section{CONVEX OPTIMIZATION PROBLEM USING LAGRANGIAN NETWORKS}

Consider the following non-linear convex programming problem with equality constraints (Xia Y., 2003):

$$
\text { Minimize } f(x) \text { subject to } A x=b
$$

where $f(x)$ is a smooth and strictly convex function, $A \in R^{m \times n}$, and $b \in R^{m}$. Remember that a functional $f: R^{n} \rightarrow R$ is strictly convex function in $X \subset R^{n}$ if, for all $x, y \in X$ and $0<\alpha<1$, we have that for all $x \neq y$ :

$f(\alpha x+(1-\alpha) y)<\alpha f(x)+(1-\alpha) f(y)$.

Consider the next Lagrangian function:

$L(x, y)=f(x)-y^{T}(A x-b)$,

where $y \in R^{m}$ is referred as the Lagrangian multiplier. $x^{*}$ is a solution to (1) if and only if there exists $y^{*} \in R^{m}$ such that $\left(x^{*}, y^{*}\right)$ satisfies the 
following Lagrangian conditions (see (Xia Y., 2003)):

$$
\begin{aligned}
& \nabla L_{x}(x, y)=\nabla f(x)-A^{T} y=0, \\
& \nabla L_{y}(x, y)=A x-b=0,
\end{aligned}
$$

where $\nabla f(x)$ is the gradient of $f(x)$ and $\left(x^{*}, y^{*}\right)$ is one stationary point of (3). Consider the next (dynamic) Lagrangian network (Xia Y., 2003):

$$
\frac{d}{d t}\left(\begin{array}{l}
x \\
y
\end{array}\right)=-\left(\begin{array}{c}
\nabla f(x)-A^{T} y \\
A x-b
\end{array}\right) .
$$

The above dynamic is said to be global convergent if, for any given initial points, all trajectories converge to an equilibrium point.

Theorem 1 (Xia Y., 2003): Assume that $\nabla^{2} f(x)$ is positive definite. Then, the Lagrangian network (4) is stable in the Lyapunov sense and is globally convergent to an equilibrium point of (4), which corresponds to a unique optimal solution of (1).

The proof of this theorem was based on Lyapunov theory to conclude that Lagrangian system (4) is stable in the Lyapunov sense; which means that all trajectories are bounded. After that, the proof is continued by proving that $x(t)$ converges to an stationary point. Finally, it was proved that this stationary point is the unique optimal solution to (1). This last part is straightforward to prove (see, (Xia Y., 2003)). The main contribution in developing proof for Theorem 1 is the use of Lyapunov theory. To facilitate further comments, below we present the stability proof given in (Xia Y., 2003) but we present a different way for the convergence of $x(t)$ to a stationary point by invoking the Barbalat's lemma.

Consider the following Lyapunov function:

$V(u)=\frac{1}{2}\|F(u)\|^{2}+\frac{1}{2}\left\|u-u^{*}\right\|^{2}$

where $u=[x, y]^{T}$,

$$
F(u)=\left(\begin{array}{c}
\nabla f(x)-A^{T} y \\
A x-b
\end{array}\right)
$$

and $u^{*}=\left[x^{*}, y^{*}\right]^{T}$ is a stationary point of the Lagrangian function. The time derivative of the Lyapunov function along the trajectories of the Lagrangian network (4) yields (Xia Y., 2003):

$\dot{V}(u)=\frac{d}{d t} V(u)$

$\leq-F(u)^{T} \nabla F(u) F(u)-\left(u-u^{*}\right)^{T} F(u)$

$\leq-\left(\nabla f(x)-A^{T} y\right)^{T} \nabla^{2} f(x)\left(\nabla f(x)-A^{T} y\right)$

$\leq 0$.

This proves stability in the Lyapunov sense of the Lagrangian network (4). Observe that $\left(\nabla f(x)-A^{T} y\right)=-\dot{x}$; so, (6) means that

$\dot{V}(u) \leq-\dot{x}^{T} \nabla^{2} f(x) \dot{x}$.

From (7), we conclude that all signals are bounded; i.e., $x, y \in L_{\infty}$, and in consequence, from (4), we have that $\dot{x}, \dot{y} \in L_{\infty}$. Integration of (7) yields:

$$
\begin{aligned}
& -V(u(0)) \leq V(u(t))-V(u(0)) \\
& \leq-\int_{0}^{t} \dot{x}^{T} \nabla^{2} f(x) x d t
\end{aligned}
$$

which implies that

$$
\left.\int_{0}^{t} \dot{x}^{T} \nabla^{2} f(x) x d t \leq V(u(0))\right)<\infty
$$

and because $\nabla^{2} f(x)$ is positive definite, then $\dot{x} \in L_{2}$. Observe that,

$$
\begin{aligned}
& \ddot{x}=\frac{d}{d t} \dot{x} \\
& \left.=-\left(\nabla^{2} f(x)\left(\nabla f(x)-A^{T} y\right)-A^{T} A x+A^{T} b\right)\right),
\end{aligned}
$$

and because $x, y \in L_{\infty}$, then $\ddot{x} \in L_{\infty}$. Invoking the well-known Barbalat's lemma (see (Krstic, 1995), Corollary A.7), we conclude that $\dot{x}(t) \rightarrow 0$ as $t \rightarrow \infty$. This implies that $x(t)$ converges to an stationary point, and in consequence, $y(t)$ converges to an stationary point too. This concludes the proof. 
We will use the above procedure in proving our main result.

Consider the next modified Lagrangian system:

$\frac{d}{d t}\left(\begin{array}{l}x \\ y\end{array}\right)=-\left(\begin{array}{c}K \nabla f(x)-A^{T} y \\ A x-b\end{array}\right)$,

where $K \in R^{n \times n}$ is symmetric and positive definite such that $K>I$. Next is our main result.

Theorem 2: Assume that $\nabla^{2} f(x)$ is positive definite. Then, the Lagrangian network (8) is stable in the Lyapunov sense and is globally convergent to an equilibrium point of (8), which corresponds to a unique optimal solution of (1).

Proof: The proof is too similar to the proof given for Theorem 1 but using $K f(x)$ instead of $f(x)$ every where. In this sense, and using the same Lyapunov function (5), we can verify that:

$\dot{V}(u) \leq-\dot{x}^{T} K \nabla^{2} f(x) \dot{x}$.

Observe that $\left(K \nabla f(x)-A^{T} y\right)=-\dot{x}$. Following the same lines used to prove Theorem 1 and invoking the Barbalat's lemma, it is easy to verify that $\dot{x}(t) \rightarrow 0$ as $t \rightarrow \infty$, meaning that $x(t)$ tends to an stationary point, so $y(t)$ does too. From (9), we can appreciate that the time derivative of the Lypunov function becomes more negative than (7) for $K>I$. This increases the speed of convergence. Proof completed.

To give a numerical example, the next quadratic optimization problem is worked out (Xia Y,,2003):

Minimize,

$\frac{1}{2}\left(\begin{array}{l}x_{1} \\ x_{2}\end{array}\right)^{T}\left(\begin{array}{cc}0.11 & 0.1 \\ 0.1 & 0.1\end{array}\right)\left(\begin{array}{l}x_{1} \\ x_{2}\end{array}\right)+\left(\begin{array}{c}-0.11 \\ 0.1\end{array}\right)^{T}\left(\begin{array}{l}x_{1} \\ x_{2}\end{array}\right)$

subject to

$\left(\begin{array}{cc}-0.5 & 0.5 \\ 0.1 & -0.1 \\ -0.2 & 0.2\end{array}\right)\left(\begin{array}{l}x_{1} \\ x_{2}\end{array}\right)=\left(\begin{array}{c}-1 \\ 0.2 \\ -0.4\end{array}\right)$, where its optimal solution is $\left(x_{1}^{*}, x_{2}^{*}\right)=(1,-1)$ (Xia Y., 2003). Simulation results are shown in Figures one and two. For Theorem 2, we used $K=\operatorname{diag}\{100,100\}$. From these Figures, we can appreciate an improvement in the settling time. In all numerical experiments we employed

$$
x(0)=\left[\begin{array}{ll}
x_{1}(0) & x_{2}(0)
\end{array}\right]^{T}=\left[\begin{array}{ll}
1 & 1
\end{array}\right]^{T},
$$

and

$$
y(0)=\left[\begin{array}{lll}
y_{1}(0) & y_{2}(0) & y_{3}(0)
\end{array}\right]^{T}=\left[\begin{array}{lll}
1 & 1 & 1
\end{array}\right]^{T} .
$$

\section{CONCLUSIONS}

We presented a slightly modification to Lagrangian networks to solve nonlinear convex programming problem with linear equality constraints. This modification was able to improve notoriously the settling time.

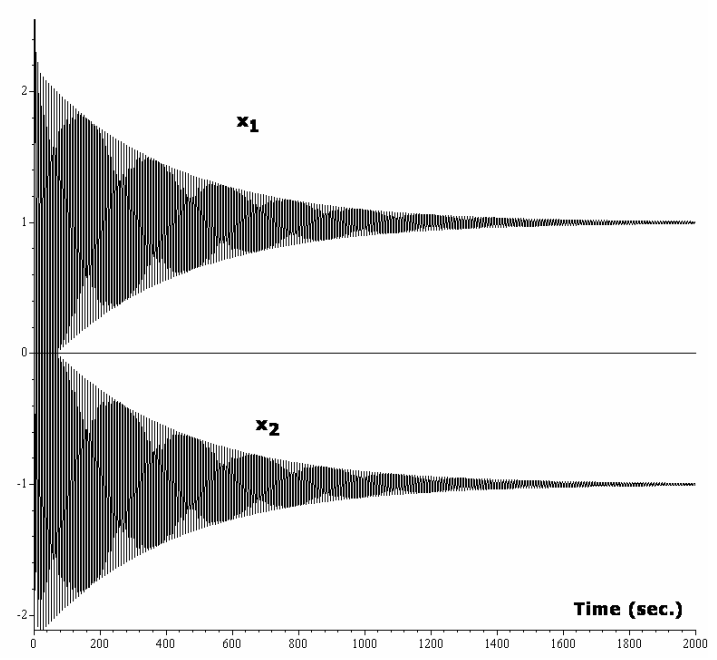

Figure 1: Simulation results using Theorem 1 


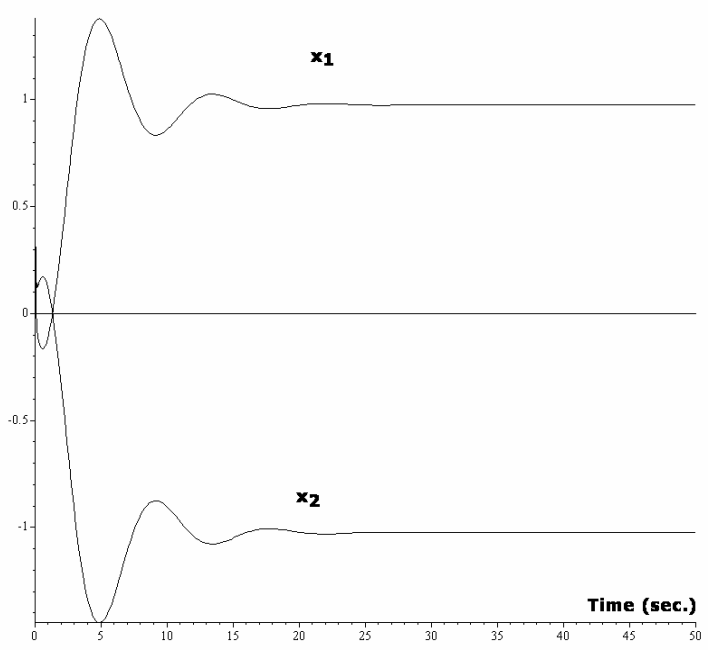

Figure 2: Simulation results using Theorem 2

\section{REFERENCES}

M. Krstic, I. Kanellakopoulos, and P. Kokotovic, 1995. Non-linear and Adaptive Control Design, John Wiley and Sons. New York.

Y. Xia, 2003. Global Convergence Analysis of Lagrangian Networks, In IEEE Transaction on Circuits and Systems I, 50 (6), 818-822, 2003. 